\title{
Multi-frequency study of the TeV blazar Markarian 421 with VLBA observations taken during 2011
}

\section{R.Lico ${ }^{* 1,2}$, M.Giroletti ${ }^{1}$, M.Orienti ${ }^{1,2}$, G.Giovannini ${ }^{1,2}$, W.Cotton ${ }^{3}$, P.G.Edwards ${ }^{4}$,

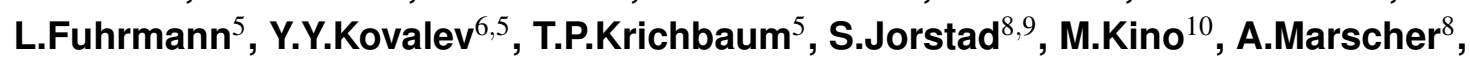 D.Paneque $^{11}$, M.A.Perez-Torres ${ }^{12}$, B.G.Piner ${ }^{13}$, K.V.Sokolovsky ${ }^{6,7}$.}

${ }^{1}$ INAF - Istituto di Radioastronomia, Bologna.

${ }^{2}$ Dipartimento di Astronomia, Università di Bologna.

${ }^{3}$ National Radio Astronomy Observatory, Charlottesville, USA.

${ }^{4}$ CSIRO Australia Telescope National Facility, Australia.

${ }^{5}$ Max-Planck-Institut für Radioastronomie, Germany.

${ }^{6}$ Astro Space Center of Lebedev Physical Institute, Russia.

${ }^{7}$ Sternberg Astronomical Institute, Moscow State University, Moscow, Russia.

${ }^{8}$ Institute for Astrophysical Research, Boston University, USA.

${ }^{9}$ Astronomical Institute, St. Petersburg State University, Russia.

${ }^{10}$ National Astronomical Observatory of Japan, Tokyo, Japan.

${ }^{11}$ Max-Planck-Institut für Physik, Germany.

${ }^{12}$ Instituto de Astrofísica de Andalucia, IAA-CSIC, Spain.

${ }^{13}$ Department of Physics and Astronomy, Whittier College, USA.

E-mail:

We present data obtained with the Very Long Baseline Array (VLBA) at twelve epochs (one observation per month from January to December 2011) at 15 and $24 \mathrm{GHz}$ for the nearby TeV blazar Markarian 421 ( $\mathrm{z}=0.031)$. We investigate the inner jet structure on parsec scales through the study of model-fit components for each epoch. The structure of Mrk 421 is dominated by a compact (FWHM about 0.13 mas) and bright component, with a one sided jet detected out to about 10 mas. We identified 5-6 components in the jet which are consistent with being stationary during the 12-month period studied here. Significant flux density variations have been detected for the core component. From our results, we estimate a viewing angle $2^{\circ}<\theta<5^{\circ}$ and a different jet velocity for the radio and the high-energy emission regions, such that the respective Doppler factors are $\delta_{r} \sim 3$ and $\delta_{\text {h.e. }} \sim 14$.

11th European VLBI Network Symposium \& Users Meeting

9-12 October 2012

Bordeaux (France)

\footnotetext{
*Speaker.
} 


\section{Introduction}

Markarian 421 (R.A. $=11^{h} 04^{m} 27.313943^{s}$, Dec. $=+38^{\circ} 12^{\prime} 31.79906 ”, J 2000$ ) is one of the nearest $(z=0.031)$ and brightest BL Lac objects in the sky. It was the first extragalactic source detected at $\mathrm{TeV}$ energies by the Cherenkov telescope at Whipple Observatory [16]. The spectral energy distribution (SED) of this object, dominated by non-thermal emission, has two smooth broad components: one at lower energies (synchrotron emission), from radio band to the soft $\mathrm{X}$ ray domain, and another at higher energies peaking at $\gamma$-ray energies (probably due to the inverse Compton scattering) [1].

At radio frequencies, Mrk 421 clearly shows a one-sided jet structure aligned at a small angle with respect to the line of sight [8]. In this work, we present new VLBA observations to study in detail the inner jet structure on parsec scales. We are able to investigate the evolution of shocks that arise in the jet, by means of the model-fitting technique. In earlier works [15, 14], the jet components show only subluminal apparent motion, which seems to be a common characteristic of TeV blazars. Thanks to accurate VLBA measurements of changes on parsec scales, we can find valid constraints on the geometry and kinematics of the jet. For this study we used the following conventions for cosmological parameters: $H_{0}=70 \mathrm{~km} \mathrm{sec}^{-1} \mathrm{Mpc}^{-1}, \Omega_{M}=0.25$ and $\Omega_{\Lambda}=0.75$, in a flat Universe.

\section{Observations}

We observed Mrk 421 throughout 2011 with the VLBA. The source was observed once per month, for a total of 12 epochs, at three frequencies: 15, 24, and $43 \mathrm{GHz}$. Here, we present the complete analysis of the whole 15 and $24 \mathrm{GHz}$ datasets. We also observed, at regular intervals, three other sources (J0854+2006, J1310+3220, and J0927+3902) used as fringe finders and calibrators for the band pass, the instrumental (feed) polarization, and the electric-vector position angle. For more detail see [9]. The analysis of the $43 \mathrm{GHz}$ and the polarization data will be the subject of two dedicated forthcoming publications (Blasi et al. in prep., Lico et al. in prep.).

\section{Results}

\subsection{Images}

A sample of two of all the images produced for each observation are shown in Fig. I, at 15 and $24 \mathrm{GHz}$ respectively. All the 12 images at each frequency show a similar structure, consisting of a well-defined and well-collimated one-sided jet structure emerging from a compact nuclear region (core-dominated source). This is the typical structure of a BL Lac object [6]. The jet extends for roughly 4.5 mas $(2.67 \mathrm{pc})^{1}$, with a position angle (PA) of $\sim-35^{\circ}$ (measured from North through East). This morphology agrees with the results of other studies of similar angular resolution [10].

\subsection{Model-fits and apparent speeds}

For each epoch, we used the model-fitting routine in DIFMAP to fit the visibility data of the source in the $(u, v)$-plane with either elliptical or circular Gaussian components. In this way,

\footnotetext{
${ }^{1} 1$ mas corresponds to $0.59 \mathrm{pc}$.
} 

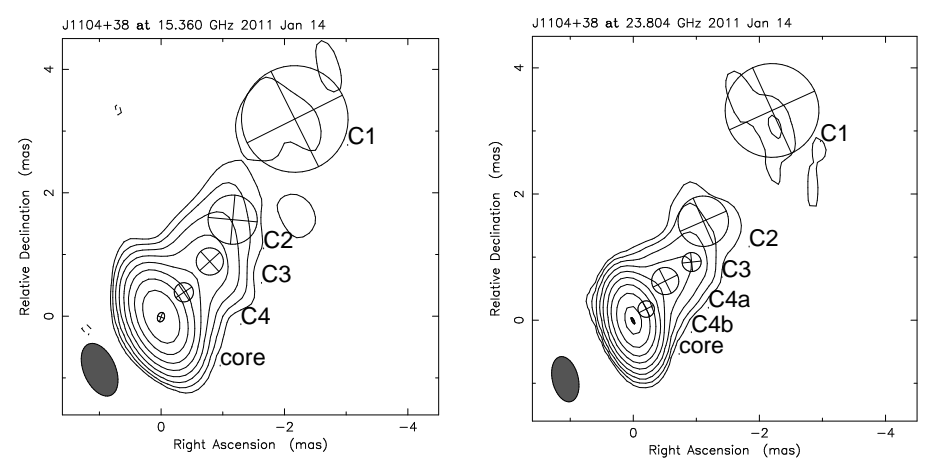

Figure 1: Images of Mrk 421 with model-fit components for the first epoch at $15 \mathrm{GHz}$ (left panel) and at $24 \mathrm{GHz}$ (right panel). Levels are drawn at $(-1,1,2,4 \ldots) \times$ the lowest contour, that is at $1.0 \mathrm{mJy} / \mathrm{beam}$ for both images, in steps of 2 . The restoring beam is shown in the bottom left corner; its size is $1.05 \times 0.65$ mas at $15 \mathrm{GHz}$ and $0.79 \times 0.47$ mas at $24 \mathrm{GHz}$.

we were able to investigate in detail the inner jet structure and its evolution. For all epochs at $15 \mathrm{GHz}$, a good fit was obtained with five Gaussian components, while at $24 \mathrm{GHz}$ we needed six components. At both frequencies, we identified the core with the brightest, innermost, and most compact feature. We label the other components $\mathrm{C} 1, \mathrm{C} 2, \mathrm{C} 3$, and $\mathrm{C} 4$, starting from the outermost (C1) to the innermost (C4). The higher angular resolution achieved at $24 \mathrm{GHz}$ resolves the second innermost $15 \mathrm{GHz}$ component ( $\mathrm{C} 4$ located at $\sim 0.45$ mas from the core) into two features ( $\mathrm{C} 4 \mathrm{~b}$ at $\sim 0.3$ mas and $\mathrm{C} 4 \mathrm{a} \sim 0.7$ mas from the core) (see Fig. $\mathrm{W}$ ).

Thanks to the extremely fine time-sampling, we identify the same component in each epoch. Overall, the components extend out to a region of about 5 mas. In this way, with a limited number of components, it was possible to analyze the proper motions and flux density levels at various times. In Fig. $\square$, we can clearly see that the data occupy well-defined regions in the radius vs. time plot, and that this behavior helps us to identify the individual components across epochs. From this plot we infer a small or no displacement for the jet components, and to verify and quantify this statement, we determined the speeds of each component by means of linear fits to the separation of the individual features from the core at different epochs. We found low values for the apparent speeds, in agreement with previous studies [e.g. 13]. The two innermost components (C4a and $\mathrm{C} 4 \mathrm{~b}$ ) are essentially stationary, with an upper limit to their separation velocity of $\sim 0.1 \mathrm{c}$. In addition $\mathrm{C} 2$ and $\mathrm{C} 3$ are consistent with being stationary, while the outermost component $\mathrm{C} 1$ has a low-significance $(1.5 \sigma)$ subluminal motion $\sim 0.3 \mathrm{c}$.

\subsection{Flux density variability and light curves}

Using the results of the model-fit technique, we analyzed the temporal evolution of the flux density for each component of the source. The brightest component represents the core; at $15 \mathrm{GHz}$, it has a mean value of around $350 \mathrm{mJy}$, which decreases along the jet until values of about $10 \mathrm{mJy}$. By comparing the flux density of each component at the various epochs, it emerges that there are no significant variations in the flux densities of the $\mathrm{C} 1-\mathrm{C} 4$ components. The flux density of each component remains roughly constant at various times within the uncertainties calculated; in any case, there is no indication of flaring activity. 


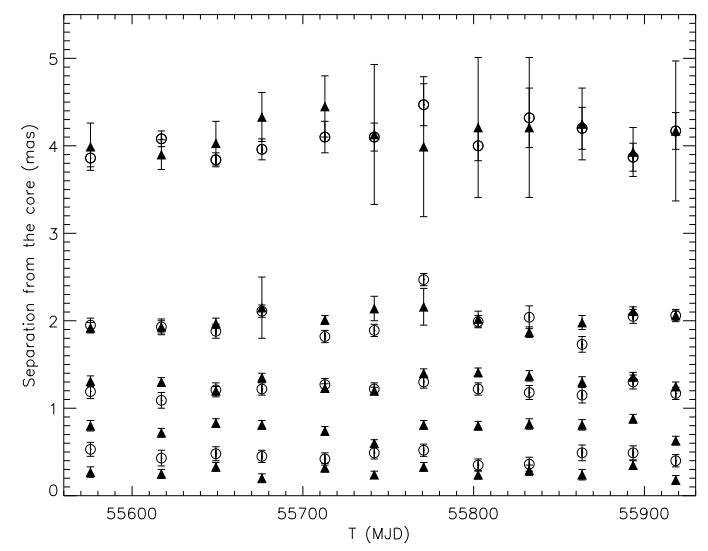

Figure 2: Results of model-fit analysis. Circles and triangles refer, respectively, to positions of the Gaussian components at 15 and $24 \mathrm{GHz}$.

In Fig. B, we show the light curves for Mrk 421 during 2011 at $15 \mathrm{GHz}$, considering the total flux density (squares), the core flux density (diamonds) and the extended emission (triangles). The light curve reveals an interesting feature: in the second part of the year (starting at MJD 55700), we clearly note a decrease in the total flux density. We can clearly see that the light curves for the core and the total flux density have the same trend, then we can argue that the core is the component responsible for the decrease. The extended region does not display any significant variations. From these considerations we can classify Mrk 421 as a core-dominated source. To further exclude calibration effects, we performed the same analysis on the three calibrators. In Fig. B, we present the light curve for the total flux density of the calibrator J1310+3220 (diamonds) in comparison with that of Mrk 421 (squares), at $15 \mathrm{GHz}$. To make a clearer comparison, all values have been normalized with the mean value of the flux density. We clearly see that the trend of the light curves for the two sources is very different. We can assert that the flux density decrease observed for the core of Mrk 421 is a real feature. Error bars were calculated by considering a calibration error of about $10 \%$ of the flux density and a statistical error equal to three times the map rms noise.

\subsection{Jet/counter-jet ratio}

We estimated the ranges of viewing angles $\theta$ and of $\beta$ from the jet/counter-jet brightness ratio. Assuming that the source has two symmetrical jets of the same intrinsic power, we used the equation

$$
\frac{B_{J}}{B_{c J}}=R=\left(\frac{1+\beta \cos \theta}{1-\beta \cos \theta}\right)^{2-\alpha}
$$

where $B_{J}$ and $B_{c J}$ are, respectively, the jet and counter-jet brightnesses and $\alpha$ represents the spectral index; we adopted the $(2-\alpha)$ exponent, since the jet is smooth and does not contain well-defined compact blobs. For the jet brightness, we used $B_{J} \sim 28.4 \mathrm{mJy} / \mathrm{beam}$, measured at $24 \mathrm{GHz}$, in the image resulting from the stacking of all the 12 epoch images, in the jet region located at $\sim 1$ mas from the core. For the counter-jet, which is not visible, we used an upper limit provided by the $3 \sigma$ rms noise level measured in the image, which resulted in $B_{c J}=0.11 \mathrm{mJy} /$ beam; this consequently yields a lower limit to both $R$ and $\beta \cos \theta$. With a value of $\alpha=-0.4$, in agreement with our spectral 

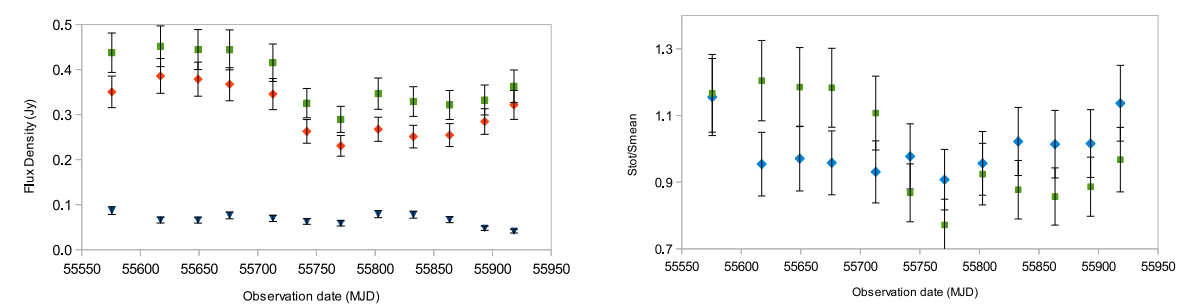

Figure 3: Left panel: light curves for Mrk421 at $15 \mathrm{GHz}$ (total flux density (squares), core flux density (diamonds) and extended emission (triangles)). Right panel: light curves of the total flux density (normalized with the mean value) $15 \mathrm{GHz}$ for Mrk 421 (squares) and the calibrator J1310+3220 (diamonds).

index images, we obtained $R>254.8$ and then $\beta \cos \theta>0.82$. Therefore, the minimum allowed jet bulk velocity is $\beta_{\min }=0.82$ (corresponding to a bulk Lorentz factor $\gamma>1.74$ ) and the maximum viewing angle is $\theta_{\max }=35.0^{\circ}$.

\section{Discussion and conclusions}

From the modeling of the SED and the study of the variability in different wavebands, generally for blazars we expect large values for the Doppler factor ${ }^{2}$; in the case of Mrk421, Gaidos et al. Gaidos et al. [3] estimated $\delta>9$, from the observed $\mathrm{TeV}$ variability time of about $30 \mathrm{~min}$ and Abdo et al. Abdo et al. [1] required a Doppler factor between 20 and 50 to reproduce the broadband SED. Surprisingly, several works [e.g. 7, 11] have reported subluminal motions, sometimes consistent with the component being stationary.

Taken together, the lack of superluminal features, the low core dominance, and the weak variability suggest a scenario in which no strong beaming is required in the radio jet. This is not uncommon in $\mathrm{TeV}$ blazars [12], but unprecedentedly firm observational support for it has been provided by our intensive campaign. Low values of the Doppler factor, e.g. $\delta \sim 3$, can reproduce the observational radio properties, including the jet brightness asymmetry.

In this scenario, it is important to understand if Doppler factors for the radio region $\left(\delta_{r}\right)$ and for the high energy region $\left(\delta_{\text {h.e. }}\right)$ have the same value or not. In the case of $\delta_{r}=\delta_{\text {h.e. }}$, combining the high value for the Doppler factor, implied by high energy observations, with stationary components implied from radio observations, we obtain unrealistic narrow viewing angles: in the case of component $\mathrm{C} 4 \mathrm{~b}$, the upper limit to the observed motion implies a viewing angle $<1.3^{\circ}$ to reproduce the observed jet/counter-jet ratio (and even smaller to agree with the high energy limits). If the jets' distribution is isotropic on the sky, the real number of misaligned sources (parent population) is incompatible with these very small values of $\theta$. On the other hand, since larger values of the viewing angle capable of reproducing the observed lack of proper motion are incompatible with the jet/counter-jet ratio, we argue that the pattern velocity cannot be representative of the bulk flow velocity; then, the low apparent speeds found imply that the proper motion of Mrk421 does not provide any information about the jet bulk velocity. We expect that the viewing angle has a value between $2^{\circ}$ and $5^{\circ}$, which is consistent with the statistical counts of low-power radio sources and

\footnotetext{
${ }^{2}$ The Doppler factor $\delta$ is calculated by the following: $\delta=\frac{1}{\gamma(1-\beta \cos \theta)}$
} 
the possibility of reaching the high Doppler factors required by SED modeling and high-energy variability.

We conclude that the Doppler factor must be different in the radio band than the $\gamma$-ray band. Since we do not expect that the viewing angle changes significantly, this leads us to the necessity of introducing a velocity structure in the jet, as previously discussed by e.g. Chiaberge et al. [2], Georganopoulos \& Kazanas [4], and Ghisellini et al. [5]. For the radio region, with a typical Lorentz factor of $\gamma \sim 1.8$, we obtain $\delta_{\mathrm{r}} \sim 3$, and for the high-energy emission region we have $\gamma \sim \delta_{\text {h.e. }} \sim 20$. Our images do not provide strong evidence in favor of either a radial or transverse velocity structure, although previous works have revealed a limb brightening in Mrk421, on both at milliarcsecond scale at $43 \mathrm{GHz}$ [11] and at $d>10$ mas at $5 \mathrm{GHz}$ [8]. This would favor the presence of a transverse velocity structure across the jet axis.

\section{References}

[1] Abdo, A. A., Ackermann, M., Ajello, M., et al., ApJ,736, 131, [arXiv:1106.1348]

[2] Chiaberge, M., Celotti, A., Capetti, A., \& Ghisellini, G., A\&A,358, 104, [arXiv:astro$\mathrm{ph} / 0003197]$

[3] Gaidos, J. A., Akerlof, C. W., Biller, S., et al., Natur,383, 319.

[4] Georganopoulos, M., \& Kazanas, D., ApJ,594, L27, [arXiv:astro-ph/0307404]

[5] Ghisellini, G., Tavecchio, F., \& Chiaberge, M., $A \& A, 432$, 401, [arXiv:astro-ph/0406093]

[6] Giroletti, M., Giovannini, G., Taylor, G. B., \& Falomo, R., ApJ,613, 752, [arXiv:astro$\mathrm{ph} / 0406255]$

[7] Giroletti, M., Giovannini, G., Feretti, L., et al., ApJ,600, 127, [arXiv:astro-ph/0309285]

[8] Giroletti, M., Giovannini, G., Taylor, G. B. \& Falomo, R., ApJ,646, 801, [arXiv:astro$\mathrm{ph} / 0604224]$

[9] Lico, R., Giroletti, M., Orienti, M., et al., A\&A,545, A117, [arXiv:1208.5853]

[10] Marscher, A. P., APh,11, 19.

[11] Piner, B. G., Pant, N. \& Edwards, P. G., ApJ,723, 1150, [arXiv:1009.2269]

[12] Piner, B. G., Pant, N. \& Edwards, P. G., ApJ,678, 64, [arXiv:0801.2749]

[13] Piner, B. G. \& Edwards, P. G., ApJ,622, 168, [arXiv:astro-ph/0412383]

[14] Piner, B. G., \& Edwards, P. G., ApJ,600, 115, [arXiv:astro-ph/0309547]

[15] Piner, B. G., Unwin, S. C., Wehrle, A. E., Edwards, P. G., Fey, A. L. \& Kingham, K. A., ApJ,525, 176, [arXiv:astro-ph/9906202]

[16] Punch, M., Akerlof, C. W., Cawley, M. F., et al., Natur,358, 477. 